\title{
Clinical characteristics, maternal and neonatal outcomes of pregnant women with SARS-CoV-2 infection in Turkey
}

\author{
Ozsurmeli $\mathrm{M}^{1}$, Terzi $\mathrm{H}^{1}$, Hocaoglu $\mathrm{M}^{2}$, Bilir RA ${ }^{2}$, Gunay $\mathrm{T}^{2}$, Unsal $\mathrm{D}^{2}$, Turgut $\mathrm{A}^{2}$, Karateke $\mathrm{A}^{2}$ \\ Department of Obstetrics and Gynecology Istanbul Medeniyet University, Goztepe Training and Research \\ Hospital, Istanbul, Turkey. dr.meryemtaskiran@gmail.com
}

\begin{abstract}
OBJECTIVES: To evaluate clinical features, laboratory test results, maternal and neonatal outcomes of pregnant patients with the Coronavirus disease (COVID-19).

BACKGROUND: COVID-19 is a highly contagious disease caused by a severe acute respiratory coronavirus 2 (SARS-CoV-2). Healthy pregnant women are more susceptible to developing COVID-19.

METHODS: We reviewed clinical data from pregnant patients with a laboratory-confirmed SARS-CoV-2, who were admitted to two tertiary care hospitals in Turkey. Demographic, clinical characteristics, laboratory test results, imaging findings, treatment received, maternal and neonatal outcomes were collected.

RESULTS: A total of 24 pregnant women were enrolled in this study. The mean maternal and gestational age was $26.9 \pm 5.37$ years and $24.15 \pm 10.61$ weeks, respectively. Cough was observed as the most common symptom ( $n=15 ; 62.5 \%)$. The lowest lymphocyte percentage was $20.83 \pm 13.05(\%)$. Nine women have delivered by Caesarean section, while one had a vaginal birth. One woman with critical COVID-19 died 2 days later postpartum. The neonate had been transferred to neonatal intensive care unit and died within 24 hours of birth.

CONCLUSION: Our findings showed that except for one patient, the clinical course of COVID-19 during pregnancy was mild. Early hospitalization of pregnant women with confirmed and suspected COVID-19, liberal testing for SARS-CoV-2, active management with a multidisciplinary team seemed to be critical to recovery (Tab. 3, Ref. 31). Text in PDF www.elis.sk

KEY WORDS: Coronavirus, COVID-19, pregnancy, SARS-Cov-2.
\end{abstract}

\section{Introduction}

The coronavirus disease (COVID-19) labelled as SARS$\mathrm{CoV}-2$, has been rapidly spreading worldwide from China to other countries in last few months and World Health Organization (WHO) declared the coronavirus infection as a pandemic (1, 2). The first case from Turkey was reported on March 13, 2020. Covid-19 is a subgroup of coronavirus family, it is genomically similar to $80 \%$ and $50 \%$ SARS-CoV and MERS-CoV, respectively (3). Rapidly, this coronavirus, namely SARS-CoV-21, has spread worldwide, leading to a serious lung inflammation, acute respiratory distress syndrome (ARDS), cardiac and renal injury, especially in patients with older age and comorbidities (diabetes mellitus, hypertension, and heart failure) (4).

${ }^{1}$ Department of Obstetrics and Gynecology, University of Health Sciences Derince Training and Research Hospital, Derince, Kocaeli, Turkey, and ${ }^{2}$ Department of Obstetrics and Gynecology Istanbul Medeniyet University, Goztepe Training and Research Hospital, Istanbul, Turkey

Address for correspondence: M. Hocaoglu, MD, Dr, Erkin cad. Istanbul Medeniyet University, Goztepe Training and Research Hospital, Kadikoy, Istanbul, Turkey.

Phone: +90.5063718935 , Fax: 0216.5666614
Evidence from the literature indicates that healthy pregnant women are more susceptible to developing COVID-19 due to their immune response that predisposes them to develop COVID-19 (5). Therefore, the cytotoxic activity of lymphocytes from healthy pregnant women was significantly reduced by progesterone at concentrations present in the serum during pregnancy (6). Many physiological changes in the maternal respiratory system make pregnant women highly susceptible to infections (7). Moreover, coronavirus infections have adverse effects not only on the mother but also on the fetus such as miscarriage, preterm birth, fetal demise, delivery of low birth-weight infants $(8,9)$. However, more studies are needed in the aspects of the impact of COVID-19 infection on maternal and neonatal outcomes.

We aimed to analyze the clinical manifestations, maternal and neonatal outcomes of COVID-19 during pregnancy in two tertiary care hospitals in Turkey.

\section{Materials and methods}

The research protocol used in this study was approved by the Istanbul Medeniyet University, Goztepe Training and Research Hospital, Clinical Studies Ethics Committee. We conducted a retrospective cohort study in pregnant women who were confirmed to have COVID-19 by a real-time quantitative reverse transcrip- 
Tab. 1. Demographic and clinical characteristics of pregnant women with COVID-19 ( $n=24)$.

\begin{tabular}{|c|c|c|}
\hline Characteristics & Mean \pm SD & $\mathrm{n}(\%)$ \\
\hline Age (y) & $26.9 \pm 5.37$ & \\
\hline Gestational age at diagnosis (wk) & $24.15 \pm 10.61$ & \\
\hline Gravidity & $2.17 \pm 3.94$ & \\
\hline Parity & $0.78 \pm 1.12$ & \\
\hline Body mass index & $28.81 \pm 4.19$ & \\
\hline \multicolumn{3}{|l|}{ Pre-pregnancy existing co-morbidities } \\
\hline Hypothyroidism & & $1(4.1)$ \\
\hline Asthma & & $1(4.1)$ \\
\hline Epilepsy & & $1(4.1)$ \\
\hline \multicolumn{3}{|l|}{ Pregnancy complications } \\
\hline Placental previa & & $1(4.1)$ \\
\hline Gestational diabetes & & $2(8.3)$ \\
\hline Preeclampsia & & $2(8.3)$ \\
\hline Placenta accreta & & $1(4.1)$ \\
\hline Number of days hospitalized & $7.30 \pm 4.43$ & \\
\hline \multicolumn{3}{|l|}{ Symptoms } \\
\hline Asymptomatic COVID-19 & & $6(25)$ \\
\hline Symptomatic COVID-19 & & $18(75)$ \\
\hline \multicolumn{3}{|l|}{ Covid-19 symptoms at first positive SARS-CoV-2 test } \\
\hline Fever & & $13(54.1)$ \\
\hline Nasal congestion & & $1(4.1)$ \\
\hline Anosmia & & $1(4.1)$ \\
\hline Cough & & $15(62.5)$ \\
\hline Shortness of breath/dyspnea & & $5(20.8)$ \\
\hline Diarrhea & & $3(12.5)$ \\
\hline Myalgia & & $13(54.1)$ \\
\hline Maximum body temperature $\left({ }^{\circ} \mathrm{C}\right)$ & $37.2 \pm 0.77$ & \\
\hline \multicolumn{3}{|l|}{ With pneumonia } \\
\hline Mild & & $1(4.1)$ \\
\hline Moderate & & - \\
\hline Severe & & $1(4.1)$ \\
\hline Respiratory co-infection & & - \\
\hline Highest respiratory rate (breaths/min) & $16.69 \pm 2.54$ & \\
\hline Lowest oxygen saturation (\%) & $96.69 \pm 2$ & \\
\hline Systolic blood pressure $(\mathrm{mmHg})$ & $115.21 \pm 8.97$ & \\
\hline Diastolic blood pressure $(\mathrm{mmHg})$ & $70.21 \pm 8.32$ & \\
\hline \multicolumn{3}{|l|}{ Pulmonary Imaging } \\
\hline US & & - \\
\hline Chest CT & & $5(20.8)$ \\
\hline CXR & & $5(20.8)$ \\
\hline Chest CT scan with ground-glass opacity & & $3(60)$ \\
\hline \multicolumn{3}{|l|}{ Treatment } \\
\hline Oseltamivir + hydroxy-chloroquine + azithromycin & & $5(20.8)$ \\
\hline Hydroxy- chloroquine + lopinavir + ritonavir +azithromycin & & $1(4.1)$ \\
\hline Hydroxy- chloroquine + azithromycin + ceftriaxone & & $1(4.1)$ \\
\hline Hydroxy- chloroquine + azithromycin & & $1(4.1)$ \\
\hline Oseltamivir +hydroxy- chloroquine + azithromycin + ceftriaxone & & $1(4.1)$ \\
\hline Hydroxy- chloroquine + lopinavir + ritonavir + ceftriaxone & & $1(4.1)$ \\
\hline Lopinavir + ritonavir + ceftriaxone & & $1(4.1)$ \\
\hline Lopinavir + ritonavir + ceftriaxone + doxycycline & & $1(4.1)$ \\
\hline Supportive therapy based on symptoms & & $5(20.8)$ \\
\hline \multicolumn{3}{|l|}{ Respiratory support } \\
\hline Nasal cannula & & $3(12.5)$ \\
\hline Mechanical ventilation & & $1(4.1)$ \\
\hline Admission to intensive care unit & & $1(4.1)$ \\
\hline Maternal mortality & & $1(4.1)$ \\
\hline
\end{tabular}

March 11, 2020 to July 1, 2020. Two authors evaluated the possibly eligible patients for concordance with the inclusion criteria for the study. All participants with a positive COVID-19 test result during any trimester of pregnancy, regardless of symptoms and age were included. Patients with negative results obtained by nasopharyngeal swabs and real-time PCR were excluded. Patients with clinical symptoms for COVID-19 (e.g., fever, cough and shortness of breath) underwent qRT-PCR to detect SARS- CoV-2 in nasopharyngeal swabs.

The demographic and clinical characteristics of pregnant women with COVID-19 were recorded. Laboratory tests included a complete blood count, aspartate transaminase (AST), alanine transaminase (ALT), C-reactive protein (CRP), D- dimer, lactate dehydrogenase (LDH), and creatine kinase. Fetal heart rate (FHR) monitoring via cardiotocography when pregnancy is $>$ 26-28 weeks of gestation, and ultrasound assessment of fetal growth and amniotic fluid volume with umbilical artery Doppler were performed. Some patients with symptoms underwent chest radiography and/or chest computed tomography (CT). Typical CT manifestations of COVID-19 such as bilateral ground-glass opacities, consolidation, an occasionally rounded morphology, and peripheral lung distribution were consistent with the guidelines of the Fleischner Society $(10,11)$. According to the WHO, patients are divided into six types: uncomplicated illness, mild pneumonia, severe pneumonia, acute respiratory distress syndrome, sepsis, and septic shock (12).

Hospital course, intensive care unit (ICU) transfer, requirement for mechanical ventilation, complications including acute respiratory distress syndrome, and disposition including discharge, maternal death were determined based on the documentation. Based on the Covid-19 national guidelines issued by the Scientific Committee of Turkish national Ministry of Health, patients were followed up and treated (13). When the women were stable, did not have shortness of breath or did not require oxygen, they were discharged and home confinement was considered.

In addition, gestational age at delivery, mode of delivery, neonatal birthweight, Apgar scores and neonatal intensive care unit admission, neonatal, pneumonia, and neonatal death were documented. tion PCR (qRT-PCR) admitted to Istanbul Medeniyet University Göztepe Training and Research Hospital and University of Health Sciences Derince Training and Research Hospital from 
152-157

\section{Statistical analyses}

Continuous variables are presented as the mean \pm SD while non-continuous variables are presented as number (percentage).

\section{Results}

A total of 24 Turkish pregnant women were enrolled in this study. All pregnancies were singleton gestations. The mean maternal age was $26.9 \pm 5.37$ years. The mean gestational age at diagnosis was $24.15 \pm 10.61$ weeks. None of the pregnant women were smoker during pregnancy. The mean body mass index of the patients was $28.81 \pm 4.19 \mathrm{~kg} / \mathrm{m}^{2}$. Of the 24 patients, $14 \%(\mathrm{n}=9)$ had an underlying health conditions including gestational diabetes $(\mathrm{n}=2)$, preeclampsia $(\mathrm{n}=2)$ asthma $(\mathrm{n}=1)$, hypothyroidism $(\mathrm{n}=$ $1)$, epilepsy $(n=1)$, placenta previa $(n=1)$, and placenta accreta $(\mathrm{n}=1)$. Detailed demographic and clinical characteristics of the participants were shown in Table 1.

All the pregnant women with COVID-19 were hospitalized. The mean number of admission days was $7.30 \pm 4.43$. Of the 24 patients, 6 (25\%) women were asymptomatic, while 18 (75\%) were symptomatic. On admission, thirteen patients $(54.1 \%)$ had fever (defined as an axillary temperature above $37.3{ }^{\circ} \mathrm{C}$ in this study). The mean maximum body temperature was $37.2 \pm 0.77^{\circ} \mathrm{C}$. Cough was observed as the most common symptom of COVID-19 $(\mathrm{n}=15$ patients, $62.5 \%$ ). Moreover, one patient developed severe COVID-19 pneumonia, while one had mild pneumonia based on the WHO definition (12). While, the mean highest respiratory rate (breaths/min) was $16.69 \pm 2.54$, lowest oxygen saturation (\%) was $96.69 \pm 2$. Five patients $(20.8)$ received a chest $x$-ray. The findings of the chest $\mathrm{x}$-rays were considered as normal. Among the patients with chest CT $(n=5$ patients, $20.8 \%), 3$ patients $(60 \%)$ had ground-glass opacity.

Laboratory testing was performed in all of the participants. While the mean lowest white blood cell (WBC) count was $7.32 \pm$ $2.58\left(10^{\wedge} 3 \mu \mathrm{L}\right)$, lowest lymphocyte percentage was $20.83 \pm 13.05$ (\%). Patients were screened at random for serum CRP considered

Tab. 2. Laboratory test results of pregnant women with COVID-19.

\begin{tabular}{lcc}
\hline Laboratory findings & Mean \pm SD & Reference ranges \\
\hline Lowest WBC count $\left(10^{3} \mu \mathrm{L}\right)$ & $7.32 \pm 2.58$ & $4-10$ \\
\hline Highest WBC count $\left(10^{3} \mu \mathrm{L}\right)$ & $8.62 \pm 3.40$ & $4-10$ \\
\hline Lowest neutrophils $\left(10^{3} \mu \mathrm{L}\right)$ & $6.49 \pm 1.56$ & $2-7$ \\
\hline Lowest lymphocyte $(\%)$ & $20.83 \pm 13.05$ & $10-50$ \\
\hline Lowest hematocrit $(\%)$ & $35.63 \pm 3.65$ & $11-15$ \\
\hline Lowest hemoglobin $(\%)$ & $11.85 \pm 1.20$ & $36-47$ \\
\hline Lowest platelets $\left(10^{3} \mu \mathrm{L}\right)$ & $190.08 \pm 55.92$ & $100-400$ \\
\hline Highest AST $($ units/L) & $23.26 \pm 12.34$ & $<31$ \\
\hline Highest ALT $($ units/L) & $14.52 \pm 9.07$ & $<37$ \\
\hline Highest CRP $(\mathrm{mg} / \mathrm{L})$ & $22.55 \pm 39.29$ & $0-0.5$ \\
\hline Highest creatinine $(\mathrm{mg} / \mathrm{dL})$ & $0.45 \pm 0.03$ & $0.57-1.11$ \\
\hline LDH $^{\mathrm{a}}$ & $250.4 \pm 74.39$ & $135-214$ \\
\hline $\mathrm{D}-$ dimer & $1372.63 \mathrm{SD} \pm 3136.85$ & $<0.50$ \\
\hline
\end{tabular}

WBC - white blood cells; AST - aspartate transaminase; ALT - alanine transaminase; CRP - C-reactive protein; $\mathrm{LDH}$-lactate dehydrogenase. ${ }^{\mathrm{a} D a t a}$ available for ten patients, ${ }^{b}$ Data available for twelve patients
Tab. 3. Neonatal outcomes for deliveries among pregnant women with COVID-19 ( $\mathbf{n = 1 0 )}$.

\begin{tabular}{lcc}
\hline Characteristics & Mean \pm SD & $\mathrm{n}(\%)$ \\
\hline Gestational age at delivery (wk) & $37.72 \pm 2.51$ & \\
\hline Days between diagnosis for COVID-19 and delivery & $16 \pm 16.5$ & \\
\hline $\begin{array}{l}\text { Mode of delivery } \\
\quad \text { Caesarean } \\
\quad \text { Vaginal delivery }\end{array}$ & $9(90)$ \\
\hline Infant birth weight (g) & & $1(10)$ \\
\hline Apgar score (1 min) & $3088 \pm 564.3$ & \\
\hline Apgar score (5 min) & $7.11 \pm 2.13$ & \\
\hline Admission to neonatal intensive care unit & $8.55 \pm 1.33$ & \\
\hline Infected with COVID-19 & $1(10)$ & \\
\hline Neonatal pneumonia & - & \\
\hline Neonatal death & & \\
\hline
\end{tabular}

as a biomarker of inflammation; the mean CRP was elevated (22.55 \pm 39.29 , reference range -0.5 ). Serum LDH level in ten patients was assessed. The mean LDH level was $250.4 \pm 4.39$. Serum levels of D-dimer were assed in 12 out of 24 patients (mean 1372.63, $\mathrm{SD} \pm 3136.85$ ). Laboratory test results of pregnant women with COVID-19 are presented in Table 2.

Five patients $(20.8 \%)$ received supportive therapy based on symptoms. The remaining nineteen patients were treated with antivirals and/or antibiotics. None of the patients received steroids. All patients received prophylactic anticoagulant therapy. Treatment protocols and distributions of treatments are shown in Table 1. There were no known cases of venous thromboembolism. Twenty-three patients recovered and were discharged; periodic follow up continued via telephone contact.

Ten of the pregnant women delivered and fourteen pregnancies were ongoing at the time of analysis. 9 women were delivered by Cesarean section, while one had a vaginal birth. All neonates had nucleic-acid testing in throat swabs and all were negative for SARS-CoV-2. One neonate required ICU and was transferred to a different hospital which has a neonatal intensive care but died within 24 hours of birth. Neonatal outcomes of the pregnant women with COVID-19 are shown in Table 3.

One COVID-19 positive patient died. She was a 39-year-old gravida with a history of gestational diabetes (diet controlled, type A1,) at 32 weeks of gestation she was admitted to hospital because of a 2- day history of myalgia, fever, dyspnea, and cough. She had a body mass index (BMI) of $40 \mathrm{~kg} / \mathrm{m}^{2}$. Her blood pressure was $120 / 90 \mathrm{~mm} \mathrm{Hg}$. Laboratory values were significant for leukocytosis and CRP was elevated (141, reference range 0-0.5). On the day of admission, CT revealed bilateral patchy groundglass features and immediately hydroxychloroquine, oseltamivir, azithromycin, and ceftriaxone were initiated. She received prophylactic anticoagulant therapy. Her SARS-CoV-2 testing on admission returned a positive result. Antepartum fetal monitoring was reassuring. She had a rapid clinical deterioration and dyspnea with acute hypoxemia $\left(\mathrm{SaO}_{2}<90 \%\right) 24$ hours later. Because of non-reassuring fetal status and severe respiratory symptoms, she underwent emergency Cesarean delivery of a viable neonate. 
The preterm newborn weighed $2000 \mathrm{~g}$, with an Apgar score of 2/6. The neonate's pharyngeal swab was SARS-CoV-2 negative on the first day. The neonate was intubated for prematurity, transferred to a different hospital which has a neonatal ICU, but died within 24 hours of birth. She was transferred to the ICU and intubated for acute respiratory distress syndrome (ARDS). Piperacillin and tazobactam were added sequentially over the next 2 days. She had markedly elevated levels of D- dimer (10000) and CRP (179 $\mathrm{mg} / \mathrm{dL}$ ). She developed severe respiratory distress, septic shock, multiple organ failure, and cardiopulmonary arrest. She died 2 days later postpartum.

\section{Discussion}

\section{Main findings of the study}

We comprehensively reviewed the maternal and perinatal outcomes of 24 pregnant women infected with COVID-19 from two tertiary care hospitals. Maternal death was observed in one woman with severe COVID-19. In the remaining cases $(n=23)$, relatively good outcomes were observed.

\section{Results in context}

The mechanisms underlying the effects of COVID-19 in pregnancy are also unknown. Nevertheless, the entry of SARS-CoV-2 is mediated by the angiotensin converting enzyme 2, ACE2 and its expression increases during pregnancy which may provide favorable conditions for SARS-CoV2 infection. In its severe stage, the pathogenesis of COVID-19 involves an uncontrolled release of pro-inflammatory cytokines, which cause systemic inflammation, ARDS, multiple organ failure, and finally lead to death (14). In the present study, maternal death occurred in a 39-year-old gravida with a BMI of $40 \mathrm{~kg} / \mathrm{m} 2$ and history of gestational diabetes at 32 weeks of gestation with a diagnosis of COVID-19 pneumonia. Unfortunately, despite appropriate treatment, our patient's symptoms had progressively worsened. Presumably, maternal obesity and gestational diabetes could be associated with severe disease in our patient. However, obesity as a risk factor for severe Covid-19 in pregnancy is known to impair lung function through both, mechanical and inflammatory pathways (15). Recent evidence revealed that the common link between metabolic diseases and SARS-CoV-2 is the inflammatory response (chronic/low-grade for metabolic diseases and acute/intense in coronavirus infection). The heat shock response (HSR), an important anti-inflammatory pathway, is decreased in patients with metabolic diseases and, consequently, may impair inflammation resolution and control in patients with SARS-CoV-2 (16).

Recent reports might alter the initial concept that pregnant women with COVID-19 were not expected to have increased risk of unfavorable outcomes $(17,18)$. Hantoushzadeh et al reported a total of 9 pregnant women with COVID-19 in their case series: 7 died and 2 survived (19). Furthermore, Takemoto et al. showed that 124 deaths of pregnant or postpartum women in Brazil, a figure which is 3.4 times higher than the total number of COVID-19-related maternal deaths reported throughout the rest of the world (18). They concluded that in Brazil, obstetric care is beset by chronic problems that can affect maternal and perinatal outcomes, such as poor-quality antenatal care, insufficient resources to manage emergency and critical care, the pandemic poses additional barriers for access to health care (18). Contrarily, the Ministry of Health has been effectively struggling to cope with SARS-CoV-2 since the early days of the pandemic in Turkey. Establishment of a competent scientific committee, identification of suspected cases by well-organized filiation teams, hospitalization of all cases of COVID- 19, administration of medications in the early stages of the disease, and specific intensive care protocols resulted in better patient outcomes than in many other countries (20). However, we reported the first case of maternal death in Turkey. In the present study, twenty-three of the remaining cases have favorable outcomes. Sahin et al. conducted a prospective cohort study in pregnant women $(\mathrm{n}=29)$ infected with COVID-19 admitted to a pandemic center in Turkey and found that no maternal death or serious morbidity occurred. On the other hand, relatively high rates of pregnancy complications $(n=7 ; 24.1 \%)$ were observed (20).

In our experience, $75 \%$ of patients were symptomatic. The common symptoms of these pregnant women at the onset of COVID-19 were fever, cough, and myalgia and the less common symptoms were anosmia and nasal congestion. Our results are consistent with results from a systematic review which has demonstrated that the most commonly reported symptoms of COVID-19 are fever, cough, dyspnea/shortness of breath, fatigue and myalgia (21). The present study showed that lymphocyte percentage was normal or low. Similarly, Wang et al. found that lymphocyte percentage was normal or decreased in pregnant patients in the early stages of COVID-19 (11). Lymphopenia associated with confirmed SARS-CoV-2 patients has also been widely reported (22). A systematic review and meta-analysis of SARS$\mathrm{CoV}-2$ infection and pregnancy revealed that the commonest laboratory abnormalities were raised CRP or procalcitonin (54.0 $\%)$, lymphopenia (34.2\%) and elevated transaminases (16.0\%). Our findings demonstrated increased level of CRP in COVID-19 positive pregnant women (23).

Coagulopathy in patients with COVID-19 is associated with an increased risk of death (24). The initial coagulopathy of COVID-19 presents with prominent elevation of D-dimer and fibrin/ fibrinogen-degradation products. Coagulation test screening, including the measurement of D-dimer and fibrinogen levels, is suggested (25). Similar to recent studies which demonstrated that raised D-Dimer levels were very common ( $\sim 85 \%)$, we found increased D-Dimer levels in COVID-19 positive pregnant women, especially in case of maternal death.

In the present study, among the 24 women with COVID-19 there were several mothers with pregnancy complications -2 women had gestational diabetes, 2 had preeclampsia, 1 had placental previa in the clinical setting of severe postpartum hemorrhage and 1 had placenta accreta. A systematic review and metaanalysis conducted by Di Mascio et al of 41 pregnant women affected by COVID-19, preeclampsia is more common than in the general population (26). There could be an association between COVID-19 and preeclampsia as it has been identified that 
SARS-CoV-2 uses the angiotensin-converting enzyme 2 receptor for cell entry $(27,28)$. Transmission of SARS-CoV-2 from the infected pregnant woman to the fetus is still controversial. Viremia is seen in only $1 \%$ of COVID-19 cases, suggesting that placental and fetal seeding might be quite rare. However, if viremia is present, the disease is more severe (29). Of the 10 neonates, we detected no virus in the nasopharyngeal swabs during the study period. Nevertheless, placenta, umbilical cord blood, or amniotic fluid were not tested to verify COVID-19 infection in the neonate. One neonate was intubated for prematurity and transferred to a different hospital which has a neonatal ICU. The neonatal death occurred on the first day of birth. Nevertheless, in any case, the cytokine storm induced by SARS-CoV-2 could result in increased morbidity and mortality among pregnant women with the potential to adversely affect the developing fetus and neonate, even in the absence of vertical viral transmission (21). However, it is important that there are no known neonatal symptoms and therefore no clinical evidence suggestive for vertical transmission, particularly when COVID-19 infection occurs later in pregnancy (26).

\section{Strengths and limitations}

This study has notable strengths which include detailed data for demographic and clinical characteristics and evaluation of data from two tertiary care hospitals. On the other hand, this study has several limitations. First, this is a small series and the results should be considered with caution. Second, the retrospective design of the study that could have influenced the interpretation of our findings. Third, 14 pregnancies were ongoing at the time of this writing, the final maternal and neonatal outcomes remain unclear.

\section{Clinical implications}

COVID-19 infection in high-risk pregnancies may result in severe maternal and neonatal outcomes such as critical respiratory failure requiring mechanical ventilation and premature termination of the pregnancy (30). It is important to known that pregnant and postpartum women admitted to the ICU with COVID-19 are at risk for maternal death (31).

In conclusion, based on the findings of the present study, except one patient, the clinical course of COVID-19 during pregnancy was mild. Early hospitalization of pregnant women with confirmed and suspected COVID-19 infection, liberal testing for SARS-CoV-2, active management with a multidisciplinary team seemed to be critical to recovery.

\section{References}

1. Yormaz B, Ergun D, Tulek B et al. The evaluation of prognostic value of acute phase reactants in the COVID-19. Bratisl Med J 2020; 121 (9): 628-633.

2. World Health Organization. WHO Director- General's opening remarks at the media briefing on COVID-1 - 3 March 2020. Available at: https://www.who.int/dg/speeches/detail/who-director-general-s-opening- remarks-at-the-media-briefing-on-covid-19-11-march-2020. Accessed June 7, 2020.

3. Tufan A, Avanoğlu Güler A, Matucci-Cerinic M. COVID-19, immune system response, hyperinflammation and repurposing antirheumatic drugs. Turk J Med Sci 2020; 50 (SI-1): 620-632.

4. Lu R, Zhao X, Li J, Niu P et al. Genomic characterization and epidemiology of 2019 novel coronavirus: implications for virus origins and receptor binding. Lancet 2020; 395: 565-574.

5. Phoswa WN, Khaliq OP. Is pregnancy a risk factor of COVID-19? Eur J Obstet Gynecol Reprod Biol 2020; S0301-2115 (20): 30433-30434.

6. Zekeres-Bartho J, Hadnagy J, Pacsa AS. The suppressive effect of progesterone on lymphocyte cytotoxicity: unique progesterone sensitivity of pregnancy lymphocytes. J Reprod Immunol 1985; 7 (2): 121-128.

7. Tan EK, Tan EL. Alterations in physiology and anatomy during pregnancy. Best Pract 456 Res Clin Obstet Gynaec 2013; 27 (6): 791-802.

8. Schwartz DA, Graham AL. Potential maternal and infant outcomes from (Wuhan) coronavirus 2019-nCoV infecting pregnant women: lessons from SARS, MERS, and other human coronavirus infections. Viruses 2020; 12: 194.

9. Alfaraj SH, Al-Tawfiq JA, Memish ZA. Middle East respiratory syndrome coronavirus (MERS-CoV) infection during pregnancy: Report of two cases \& review of literature. J Microbiol Immunol Infect 2019; 52 : 501-503.

10. Rubin GD, Ryerson CJ, Haramati LB et al. The Role of Chest Imaging in Patient Management during the COVID-19 Pandemic: A Multinational Consensus Statement from the Fleischner Society. Chest 2020; 158 (1): 106-116.

11. Wang $\mathbf{Z}$, Wang $\mathbf{Z}$, Xiong G. Clinical characteristics and laboratory results of pregnant women with COVID-19 in Wuhan, Int J Gynaecol Obstet 2020; 10.1002/ijgo.13265.

12. World Health Organization (2020) Clinical management of severe acute respiratory infection when COVID-19 is suspected. https: //www. who.int/publications-detail/clinical-management-of-severe-acute-respiratory-infection-when-novel-coronavirus- (ncov)-infection-is-suspected.

13. Covid-19 (sars-cov-2 enfeksiyonu) rehberi bilim kurulu çalişmasi T.C. Sağlik Bakanliği 14 Nisan 2020, Ankara

14. Muyayalo KP, Huang DH, Zhao SJ et al. COVID-19 and Treg/ Th17 imbalance: Potential relationship to pregnancy outcomes Am J Reprod Immunol 2020; e13304.

15. Dixon AE, Peters U. The effect of obesity on lung function. Expert Rev Respir Med 2018; 12 (9): 755-767.

16. Krause M, Gerchman F, Friedman R. Coronavirus infection (SARSCoV-2) in obesity and diabetes comorbidities: is heat shock response determinant for the disease complications? Diabetol Metab Syndr 2020; $12: 63$.

17. Takemoto MLS, Menezes MO, Andreucci CB et al. Maternal mortality and COVID-19 J Matern Fetal Neonatal Med 2020; 1-7.

18. Takemoto MLS, Menezes MO, Andreucci CB et al. The tragedy of COVID-19 in Brazil: 124 maternal deaths and counting Int J Gynaecol Obstet 2020; 10.1002/ijgo.13300.

19. Hantoushzadeh S, Shamshirsaz AA, Aleyasin A et al. Maternal death due to COVID-19. Am J Obstet Gynecol 2020; 223 (1): 109.e1-109.e16.

20. Sahin D, Tanacan A, Erol SA et al. A pandemic center's experience of managing pregnant women with COVID-19 infection in Turkey: A prospective cohort study. Int J Gynaecol Obstet 2020; 10.1002/ijgo.13318. 
21. Verma S, Carter EB, Mysorekar IU. SARS-CoV2 and pregnancy: an invisible enemy? Am J Reprod Immunol 2020; e13308.

22. Wang D, Hu B, Hu C et al. Clinical Characteristics of 138 Hospitalized Patients With 2019 Novel Coronavirus- Infected Pneumonia in Wuhan, China. JAMA 2020; 323 (11): 1061-1069.

23. Khalil A, Kalafat E, Benlioglu $\mathbf{C}$ et al. SARS-CoV-2 infection in pregnancy: a systematic review and meta-analysis of clinical features and pregnancy outcomes. E Clinical Medicine, 2020, 100446.

24. Levi M, Thachil J, Iba T et al. Coagulation abnormalities and thrombosis in patients with COVID-19. Lancet Haematol 2020; 7 (6): e438-e440.

25. Connors JM, Levy JH. COVID-19 and its implications for thrombosis and anticoagulation. Blood 2020; 135 (23): 2033-2040.

26. Di Mascio D, Khalil A, Saccone G et al. Outcome of Coronavirus spectrum infections (SARS, MERS, COVID 1 -19) during pregnancy: a systematic review and meta-analysis Am J Obstet Gynecol MFM 2020; 2 (2): 100107.
27. Pereira A, Cruz-Melguizo S, Adrien M et al. Clinical course of coronavirus disease-2019 in pregnancy. Acta Obstet Gynecol Scand 2020; 99 (7): 839-847.

28. Coronaviridae Study Group of the International Committee on Taxonomy of Viruses. The species Severe acute respiratory syndrome-related coronavirus: classifying 2019-nCoV and naming it SARS-CoV-2. Nat Microbiol 2020; 5 (4): 536-544.

29. Ovali F. SARS-CoV-2 Infection and the Newborn. Front Pediatr 2020; 8: 294.

30. Kolkova Z, Bjurström MF, Länsberg JK et al. Obstetric and intensive-care strategies in a high-risk pregnancy with critical respiratory failure due to COVID-19: A case report. Case Rep Womens Health 2020; 27: e00240.

31. Blitz MJ, Rochelson B, Minkoff $\mathbf{H}$ et al. Maternal mortality among women with coronavirus disease 2019 admitted to the intensive care unit. Am J Obstet Gynecol 2020; S0002-9378 (20): 30636-0.

Received August 28, 2020. Accepted September 16, 2020. 